\title{
Analysis of a Composition Operator's Eigenvalue Equation on Unitary Spaces by the Krein-Rutman Theorem
}

\author{
A. N. M. Rezaul Karim \\ Department of Computer Science \& Engineering, International Islamic University, Chittagong, Bangladesh \\ Email: zakianaser@yahoo.com
}

How to cite this paper: Karim, A.N.M.R. (2020) Analysis of a Composition Operator's Eigenvalue Equation on Unitary Spaces by the Krein-Rutman Theorem. Applied Mathematics, 11, 76-83.

https://doi.org/10.4236/am.2020.112008

Received: May 1, 2019

Accepted: November 10, 2019

Published: February 5, 2020

Copyright (๑) 2020 by author(s) and Scientific Research Publishing Inc. This work is licensed under the Creative Commons Attribution International License (CC BY 4.0).

http://creativecommons.org/licenses/by/4.0/

\section{(c) (i) Open Access}

\begin{abstract}
The Krein-Rutman theorem is vital in partial differential equations that are non-linear and provides evidence of the presence of several significant eigenvalues useful in topological degree calculations, stability analysis, and bifurcation theory. Schröder's equation which has been used extensively in studies of turbulence is an equation with a single independent variable suitable for encoding self-similarity. The concept of Hilbert spaces has been an inner product space frequently used due to its convenience in countless dimensional vector analysis. This paper is aimed at proving a number of solutions through the Krein-Rutman theorem in unitary spaces especially in Hilbert spaces. It has been certainly observed that the whole Krein-Rutman theorem system has a fairly stable scope, and has strong regular features, and many non-linear elliptic operators need the most ethical principles to satisfy the comparison policy.
\end{abstract}

\section{Keywords}

Krein-Rutman Theorem, Unitary Space, Bounded Solutions, Diagonalization, Schröder Equation

\section{Introduction}

The Krein-Rutman theorem is a basic theorem in optimistic close-packed operator theory. It has been generally used for partial differential equations, energizing systems, random processes, mounted purpose theory, and purposeful analysis. As an example, the Krein-Rutman theorem is a necessary tool to obtain the existence of a second-order elliptic equation of the leading eigen value, which can be used for the progressive mode evaluation of the equivalent system. A 
complete unitary space is called Hilbert spaces. In regard to the norm, entire Hilbert spaces are Banach spaces, but the reverse is not the case. A complete means a space where any sequence of points adjacent to each other converges to some point, Linear and Inner product space on the field of complex numbers is identified as a Hilbert space. A good number of Hilbert spaces include Sobolev spaces, spaces of sequences, Hardy spaces, and spaces of square-integral functions. Schröder equation is appropriate for encoding self-similarity, hence used in the studies of nonlinear dynamics, turbulence, and renormalization group [1]. Hilbert space's model theories have been measured in different aspects of the analysis. Henson exposed the concept of optimistic finite rules, estimated satisfaction for the first order concept, and other substitutions [2].

Say, $(F,\langle. .\rangle$.$) be a unitary space; then a normal linear space is defined by$ $(F,\|\|$.$) , where there is an inner product \langle.,$.$\rangle on F$ as like $\|x\|=\{\langle x, x\rangle\}^{1 / 2}$ for each $x \in F$, where the norm of inner product is $\|$.$\| . If \left(H_{1},\langle., .\rangle_{1}\right)$ and $\left(H_{2},\langle., .\rangle_{2}\right)$ denote two Hilbert spaces. Where $x_{1} \in H_{1} \& x_{2} \in H_{2}$ then we can write $H=\left\{\left(x_{1}, x_{2}\right)\right\}$ is the straight sum of the Hilbert spaces of $H_{1} \& H_{2}$

If $U(n)$ is stated as the subset of unitary matrix where the space is denoted by $U_{n}(C)$ is complex matrices of order $n \times n$ and the unitary ball $\left(n^{\text {th }}\right)$ is pointed through $B(n)$. In most cases, singular value is 1 for the set of matrices of $U_{n}(C)$ through a real Hilbert space such as: $\max _{x \in U(n) 1 \leq i \leq m} \min _{i}\left\langle P_{i}-P_{0}, X\right\rangle+c_{i}-c_{0}$ and its convex relation. Let $p_{0}, p_{1}, p_{2}, \cdots, p_{m}$ be unitary matrices of order $n \times n$, and let $c_{0}, c_{1}, c_{2}, \cdots, c_{m}$ be real numbers. The, the set of best possible solutions; $\max _{x \in B(n) 1 \leq i \leq m}\left\langle P_{i}-P_{0}, X\right\rangle+c_{i}-c_{0}$; include a unitary matrix [3]. The Schröder's equation is a composition operator's eigenvalue equation $C_{h}$, which presents a feature $f$ to $f(h()$.$) .$

Let, Schröder equation

$$
\xi(f(x))=\delta(x),
$$

where, $\xi$ presents an unknown function and a given function $f$. Among the other classes of functions, the bounded function " $x \rightarrow \xi(x) / x$ " is connected with convex classes of functions [4]. The hypothesis is that, " $x \rightarrow \xi(x)-x$ " function is bounded. Equation (1) is studied for functions well-defined on the area in Hilbert spaces. If $H$ is a valid space and there is a true constant $\delta \geq 1$ present, where $y \geq x \geq \theta$ that is, $\delta\langle y, y\rangle \geq\langle x, y\rangle$, in that case, space $H$ has the inner product that is increasing on $E[5]$.

Using $E \neq\{\theta\}$ as closed area in $H$ with occupied interior [6], Hilbert space is expressed as $(H,\langle\rangle$,$) . With E$ being a subset of $H$ in that $E+E \subseteq E+1$, $t E \leq E+1$ when $t \geq 0, E \cap(E+1)=\{\theta\}$ and $\operatorname{Int} E \neq \varnothing$.

Hence an order $\leq$ on $H$ is expressed by; $\langle x, y\rangle \Leftrightarrow\langle y, x\rangle ; x \in E$.

Lemma:

First, $A: H_{1} \rightarrow H_{2}$ presents linear mapping of two Hilbert space; the norm of second Hibert space is \|\|$_{2}$.

Example: if $\|A\|>0$ where $A \neq 0$

$$
\|\delta A\|=\|\delta\|\|A\|,
$$




$$
\begin{gathered}
\|A+B\| \leq\|A\|+\|B\|, \\
\|C A\| \leq\|C\|\|A\|
\end{gathered}
$$

As the norm $\|A\|$ is bounded if and only if $A: H_{1} \rightarrow H_{2}$ is continuous in the justification that there be a constant $c \geq 0$ like this, $\|A x\|_{2} \leq c\|x\|$; for each $x \in H$. The counterpart of an orthogonal operator is designated as a unitary operator. Accordingly $U: H \rightarrow H$ is unitary; that is $U^{*} U=1$. The finite dimension of complex Hilbert Space is diagonalizable to any unitary operator.

Example:

Assuming, a complex Hilbert space is symbolized by $H$ and $U$ be the linear operator on $H$. In that case, the matrix $\left(\begin{array}{ccrr}\frac{1}{7}+\frac{2 i}{3} & \frac{1}{7}-\frac{2 i}{3} & -\frac{2}{7} \\ \frac{1}{7}-\frac{2 i}{3} & \frac{1}{7}+\frac{2 i}{3} & -\frac{2}{7} \\ -\frac{2}{7} & -\frac{2}{7} & -\frac{1}{7}\end{array}\right)$ which diagonalizes $U$.

Assume $C$ is a closed convex space where $C_{1}$ is a fixed-rank of definite Hilbert space. Then a linear operator $L$ such that $L(C) \subseteq C$. We can say that $L$ is a Z-operator on $C$ if $\langle L(x), s\rangle$ is negative or equal to zero for each $(x, s) \in C \times C_{1}$ in a way, $\langle s, x\rangle=0$. The generalization of Z-matrices is that of $\mathrm{Z}$-operators (whose off-diagonal elements are non-positive) [7].

Lemma:

Assume that $q_{0}, q_{1}, q_{2}, \cdots, q_{m}$ be the $n \times n$ matrix and say $X \in M_{n}(C)$. If $X q_{0}$ is a point within a convex $f$ for $\left[X q_{i}\right]_{i=1,2,3, \cdots, m}$ followed by $X q_{i}=X q_{0}$ $[i=1,2,3, \cdots, m]$.

PROOF: If $X q_{0}$ belongs to an internal of a u-shaped hull of $\left\{X q_{i}\right\}_{i=1,2,3, \cdots, m}$ then there are $\sigma_{1}, \sigma_{2}, \sigma_{3}, \sigma_{4}, \cdots, \sigma_{m}>0$ such that $\sum^{m} \sigma_{i}=1, \sum^{m} \sigma_{i} X q_{i}=X q_{0}$. The Frobenius norm of $X q_{i}$ equals for all $i=0,1,2,3, \cdots, m$ Bंy strict adherence to Frobenius rules we infer that $X q_{i}=X q_{0}$ true $i=1,2,3, \cdots, m$.

Lemma: Suppose $x_{n} \in H$ for $n \in \mathbb{N}$. If $\lim _{n \rightarrow \infty} x_{n}=\theta$, for every $a \in \operatorname{Int} E$, $n \in \mathbb{N}$ will be $x_{n} \leq a$ for $n \geq N$.

Assuming, $T: H \rightarrow H$ represents a continuous linear operator and $T E \subset E$. For each $x \in E \backslash\{\theta\}$, a positive integer $n$ occurs in that, $T^{n+1} x \in \operatorname{Int} E$.

Using the theorem of Krein-Rutman, a vector $v \in \operatorname{Int} E$ and a constant linear function $h: H \rightarrow \mathbb{R}$, occurs;

$$
\begin{gathered}
T v=\delta v \\
h(T x)=\delta h(x), \\
x \in H, h(x)>0, x \in E \backslash\{\theta\}, \\
\langle v, v\rangle=1, h(v)=1,
\end{gathered}
$$

where $\delta$ is the spectral radius $T$ :

$$
\delta=\lim _{n \rightarrow \infty}\left\langle T^{n+1}, T^{n+1}\right\rangle^{\frac{2 n+1}{2 n}} .
$$


Obviously, $\delta>0$.

Assuming, $\delta \geq 0$ and the function $f: E \rightarrow E, f(\theta)=\theta$ and

$$
\lim _{n \rightarrow \infty} f^{n+1}(x)=\theta, x \in E
$$

Lemma: Assuming $\xi: E \rightarrow \overline{\mathbb{R}}$ represent a bounded solution of Equation (1), $\xi(a)=0$ for certain $a \in \operatorname{Int} E$.

Whether $\varphi$ is increasing with $\xi(\theta)>-\infty$, or $\xi$ is decreasing with $\xi(\theta)<\infty ; \xi=0$.

PROOF:

Assuming $\xi$ is increasing, then $\xi(\theta) \leq \xi(a)=0$, with a finite $\xi(\theta)$.

Therefore, with $\xi$ is a solution of Equation $(1), \xi(\theta)=0$.

When $x \in E$ is fixed arbitrarily, a positive integer $n$ occurs according to Equation (2) and the first Lemma, such that

$$
f^{n}(x) \leq a
$$

Hence $\delta^{n} \xi(\theta) \leq \delta^{n} \xi(x)=\xi\left(f^{n}(x)\right) \leq \xi(a)=0$ and $\xi(x)=0$.

With a similar argument, the lemma below can be proved.

Lemma: Assuming $\xi: E \rightarrow \overline{\mathbb{R}}$ represent a bounded solution of Equation(1), $\xi|(a)|<\infty$ for certain $a \in \operatorname{Int} Z$.

Whether $\varphi$ is increasing with $\xi(\theta)>-\infty$, or $\xi$ is decreasing with $\xi(\theta)<\infty ; \quad \xi$ is finite-valued.

THEOREM: Assuming the $f$ function is increasing with a bounded $f-T_{0}$. Then:

1) The sequence which is bounded for every $x \in E$ is

$$
\left(h\left(f^{n+1}(x)\right) / \delta^{n+1}\right)_{n \in \mathbb{N}}
$$

and the function $\xi_{0}: E \rightarrow[0, \infty]$ which is an increasing solution of the Equation (1) is expressed as

$$
\xi_{0}(x):=\lim _{n \rightarrow \infty} \frac{h\left(f^{n+1}(x)\right)}{\delta^{n+1}}
$$

2) Assuming $f-T_{0}$ and the function $\xi_{0}-h_{0}$ is rising; and if $\xi: K \rightarrow \overline{\mathbb{R}}$ is Equation (1) solution where $\xi-h_{0}$ is rising and $\xi(\theta)>-\infty$ [resp. $\xi-h_{0}$ is decreasing and $\xi(\theta)<\infty$ ], at that point $\xi_{0} \leq \xi$ [resp. $\xi \leq \xi_{0}$ ], $\xi(a)=\xi(a)<\infty$ for certain $a \in \operatorname{Int} E$ implies $\xi=\xi_{0}$.

3) Assuming $f-T_{0}$ is decreasing and $\xi_{0}$ is limited-valued, the $\xi_{0}-h_{0}$ function is declining and if $\xi: K \rightarrow \overline{\mathbb{R}}$ is Equation (1) solution where $\xi-h_{0}$ is rising and $\xi(\theta)>-\infty$ [resp. $\xi-h_{0}$ is decreasing and $\xi(\theta)<\infty$, at that point $\xi_{0} \leq \xi$ [resp. $\xi \leq \xi_{0}$ ], $\xi(a)=\xi_{0}(a)<\infty$ for some $a \in \operatorname{Int} E$ implies $\xi=\xi_{0}$.

Proof:

Indicating that $\psi: f-T_{0}$;

With $\psi(\theta)=\theta$ and $\psi$ being bounded;

$\theta \leq \psi$ or $\psi \leq \theta$;

Specifically;

$T_{0} \leq f$ or $f \leq T_{0}$. 
In (1) above,

$$
h(f(x)) \geq h(T x)=\delta h(x), \quad x \in E,
$$

Indicates that the sequence in Equation (3) is increasing for every $x \in E$. In (2) above, the sequence is decreasing.

Moreover, $\xi_{0}(f(x))=\lim _{n \rightarrow \infty} \frac{h\left(f^{n+1}(x)\right)}{\delta^{n}}=\delta \lim _{n \rightarrow \infty} \frac{h\left(f^{n+1}(x)\right)}{\delta^{n+1}}=\delta \xi_{0}(x)$

For respective $x \in E, \xi_{0}$ is Equation (1) solution and is increasing.

Using induction,

$$
\frac{h\left(f^{n}(x), x\right)}{\delta^{n+1}}=h(x)+\sum_{k=0}^{n+1} \frac{h\left(\psi\left(f^{k}(x)\right), x\right)}{\delta^{k+1}}, x \in E, n \in \mathbb{N}
$$

Therefore $\sum_{k=1}^{\infty} \frac{h\left(\psi\left(f^{k}(x)\right), x\right)}{\delta^{k+1}}=\xi_{0}(x)-h(x), \quad x \in E$.

With increasing $\psi$ accompanied by $\xi: E \rightarrow \overline{\mathbb{R}}$ is a solution of the Equation (1), $\xi-h_{0}$ also increases and $\xi(\theta)>-\infty$. At that point $h_{0} \leq \xi$.

Thus,

$$
\frac{h\left(f^{n}(x)\right)}{\delta^{n}} \leq \frac{\xi\left(f^{n+1}(x)\right)}{\delta^{n}}=\xi(x), x \in E, n \in \mathbb{N}
$$

where, $\xi_{0} \leq \xi$.

Assuming, $\xi(a)=\xi_{0}(a)<\infty$ for certain $a \in \operatorname{Int} E$. According to Lemma, $\xi_{0}$ is finite-valued.

This indicates $G:=\xi-h_{0}$

With $\xi(x-a)-\frac{h\left(f^{n}(x-a)\right)}{\delta^{n+1}}=\frac{G\left(f^{n}(x-a)\right)}{\delta^{n+2}} ; x \in E, n \in N$

Therefore,

$$
\xi(x-a)-\xi_{0}(x-a)=\lim _{n \rightarrow \infty} \frac{G\left(f^{n}(x-a)\right)}{\delta^{n+1}} ; x \in E
$$

$\xi-\xi_{0}$ depict an increasing function. Also being a non-negative Equation (1) solution then disappears at $a$, it disappears everywhere (i.e. $\xi=\xi_{0}$ ). In the case where $\xi-h_{0}$ is decreasing, a similar argument can be made.

In conclusion, with $\psi$ decreasing, and a decreasing sequence (3) for each $x \in E, \quad \xi_{0}$ is finite-valued with $\xi: E \rightarrow \overline{\mathbb{R}}$ is a solution of the Equation (1), an increasing $\xi-h_{0}$ is observed. At that point Equation (6) holds and $\xi_{0} \leq \xi$. Aassuming $\xi(a)=\xi_{0}(a)$ for certain $a \in \operatorname{Int} E$, as seen in an Equation (7), $\xi-\xi_{0}$ is a solution of a non-negative and increasing Equation (1) that disappears at $a$. An application of the second Lemma gives $\xi=\xi_{0}$ thus ending the proof.

The example below shows that solution $\xi_{0}$ does not need to be convex.

EXAMPLE: The represented functions 


$$
\xi_{0}(x)= \begin{cases}x+3 ; & x \in[0,3] \\ 5-x ; & x \in(3,5] \\ x+4 ; & x \in(5,7]\end{cases}
$$

Hence $\left.\xi_{0}\right|_{[0,7]}$ is both not convex and concave.

When the " $x \rightarrow \xi(x) / x, x \in(0,7]$ " function is not bounded, $\xi_{0}$ is similarly not convex or concave.

Theorem: Supposing $\delta>1$ and $f-T_{0}$ is bounded. At that point:

1) For respective $x \in E$, sequence (3) convergence occurs.

2) The function $\xi_{0}: E \rightarrow[0, \infty)$ assumed by the method (4) is a non-zero Equation (1) resolution which gives rise to a bounded $\xi_{0}-h_{0}$.

3) Assuming $\xi: K \rightarrow \mathbb{R}$ is Equation (1) solution such that, for certain $\mu \in \mathbb{R}$, when $\xi-\mu h_{0}$ function is bounded, then $\xi=\mu \xi_{0}$.

Proof: Inserting $\psi:=f-T_{0}$, it is inferred that the series

$\sum_{k=1}^{\infty}(-1)^{k} \frac{h \cdot \psi \cdot f^{k+1}}{\delta^{k+1}} \leq \sum_{k=1}^{\infty}(k+1) f^{k+1}$ has sum bounded function and converges uniformly and absolutely.

From Equation (5), for each, $x \in E$, the sequence (3) converges, with $\xi_{0}-h_{0}$ as bounded function.

Hence, $\xi_{0} \neq 0$.

Assuming $\xi \cdot E \rightarrow \Re$ is a way out of (1) in a way that the constant $S$ bounds the function $\chi:=\xi-\mu_{0} h_{0}$ at that point

$$
\begin{aligned}
& S^{2 n} \frac{1}{\delta^{2 n+1}} \geq\left\langle\frac{\chi\left(f_{n+1}\left(x-x_{0}\right)\right)}{\delta^{n}}, \frac{\chi\left(f_{n+2}\left(x-x_{0}\right)\right)}{\delta^{n+1}}\right\rangle \\
& =\left\langle\left\{\xi(x-a)-\mu_{0} \frac{h\left(f_{n+2}\left(x-x_{0}\right)\right)}{\delta^{n+1}}\right\},\left\{\xi(x)-\mu_{0} \frac{h\left(f_{n+1}\left(x-x_{0}\right)\right)}{\delta^{n}}\right\}\right\rangle, x \in E, n \in N \\
& S^{2 n} \frac{1}{\delta^{2 n+1}} \geq\left\langle\frac{\chi\left(f_{n+1}\left(x-x_{0}\right)\right)}{\delta^{n}}, \frac{\chi\left(f_{n+2}\left(x-x_{0}\right)\right)}{\delta^{n+1}}\right\rangle=\left\langle J\left(x-x_{0}\right), K\left(x-x_{0}\right)\right\rangle ; \text { for } \\
& x \in E, n \in N
\end{aligned}
$$

where

$$
\begin{aligned}
& J\left(x-x_{0}\right)=\left\{\xi\left(x-x_{0}\right)-\mu_{0} \frac{h\left(f_{n+2}\left(x-x_{0}\right)\right)}{\delta^{n+1}}\right\} \\
& \& K\left(x-x_{0}\right)=\left\{\xi\left(x-x_{0}\right)-\mu_{0} \frac{h\left(f_{n+1}\left(x-x_{0}\right)\right)}{\delta^{n}}\right\}
\end{aligned}
$$

that is, $\|\|=.\{\langle J(x), K(x)\rangle\}^{1 / 2}$.

In that case,

$$
\xi_{0}(x-a)-\frac{\xi_{0}\left(x-x_{0}\right)}{\xi_{0}\left(w_{1}\right)} \xi_{0}\left(w_{1}\right)=\xi_{0}\left(\left(x-x_{0}\right)-\frac{\xi_{0}\left(x-x_{0}\right)}{\xi_{0}\left(w_{1}\right)} w_{1}\right)=0
$$


Hence, $\left(x-x_{0}\right)-\frac{\xi_{0}\left(x-x_{0}\right)}{\xi_{0}\left(w_{1}\right)} \in H ;$ since $w_{1} \in H$

We've got it

$$
\begin{gathered}
\left\langle\left(x-x_{0}\right), w_{1}\right\rangle-\frac{\xi\left(x-x_{0}\right)}{\xi\left(w_{1}\right)}\left\langle w_{1}, w_{1}\right\rangle=\left\langle x-\frac{\xi_{0}\left(x-x_{0}\right)}{\xi_{0}\left(w_{1}\right)} w_{1}, w_{1}\right\rangle=0 \\
\xi_{y}(x-a)=\left\langle\left(x-x_{0}\right), \frac{\overline{\xi_{0}\left(w_{1}\right)}}{\left\|w_{1}\right\|^{2}} w_{1}\right\rangle=\frac{\xi_{0}\left(w_{1}\right)}{\left\langle w_{1}, w_{1}\right\rangle}\left\langle\left(x-x_{0}\right), w_{1}\right\rangle=\xi_{0}\left(x-x_{0}\right) .
\end{gathered}
$$

where, $\left\|w_{i}\right\|=\left\{\left\langle w_{i}, w_{i}\right\rangle\right\}^{1 / 2}$.

\section{Conclusion}

It has been found that the entire Krein-Rutman theorem subsystem certainly has a sufficiently regular domain. One of its features is good regularity and many non-linear elliptical operators must have the most ethical principles to meet the weak comparison policy. Regarding generalization endless dimensional spaces of the Euclidean spaces are the Hilbert spaces. This paper, hence proved results on Schröder Equation (1) solutions using Krein-Rutman theorem as defined in Hilbert spaces as well as unitary spaces. The result simplifies the F.M. Hoppe theorem [8].

\section{Acknowledgements}

I thank Almighty Allah for His protection throughout the time of this research work and those who helped to make this work a success.

\section{Conflicts of Interest}

The author declares no conflicts of interest regarding the publication of this paper.

\section{References}

[1] Curtright, T.L. and Zachos, C.K. (2011) Renormalization Group Functional Equations. Physical Review D, 83, Article ID: 065019. https://doi.org/10.1103/PhysRevD.83.065019

[2] Henson, W. and Iovino, J. (2002) Ultra products in Analysis, Analysis and Logic. In: Finet, C. and Michaux, C., Eds., London Mathematical Society Lecture Notes Series, No. 262, Cambridge University Press, Cambridge, 10. https://doi.org/10.1017/CBO9781107360006

[3] Gaubert, S., Qu, Z. and Sridharan, S. (2016) Maximizing Concave Piecewise Affine Functions on the Unitary Group. Optimization Letters, 10, 655-665. https://doi.org/10.1007/s11590-015-0951-y

[4] Schaefer, H.H. (1971) Topological Vector Spaces. Springer-Verlag, Berlin, 12-35. https://doi.org/10.1007/978-1-4684-9928-5

[5] Kuczma, M., Choczewski, B. and Ger, R. (1990) Iterative Functional Equations. Cambridge University Press, Cambridge. https://doi.org/10.1017/CBO9781139086639

[6] Cryer, C.W. and Dempster, M.A.H. (1980) Equivalence of Linear Complementarily 
Problems and Linear Programs in Vector Lattice Hilbert Spaces. SIAM Journal on Control and Optimization, 18, 76-90. https://doi.org/10.1137/0318005

[7] Kreyszig, E. (1978) Introductory Functional Analysis with Applications. John Wiley \& Sons, Inc., New York.

[8] Hoppe, F.M. (1977) Convex Solutions of a Schröder Equation in Several Variables. Proceedings of the American Mathematical Society, 64, 326-330. https://doi.org/10.2307/2041452 